\title{
PROCEDIMIENTOS DE ATENUACIÓN EN LOS ARTÍCULOS DE INVESTIGACIÓN CIENTÍFICA: LAS DIRECTRICES COGNITIVAS EN ESPAÑOL
}

\section{MITIGATION IN RESEARCH ARTICLES: COGNITIVE DIRECTIVES IN SPANISH}

\author{
Maikel Chao Parapar \\ Universidade da Coruña \\ Grupo HISPANIA \\ maikel.dcp@gmail.com
}

Recibido: $19 / 12 / 2016$

Aceptado: 19/03/2017

\begin{abstract}
Resumen
Entre las características del discurso científico se encuentra el empleo de una serie de recursos que se engloban bajo la etiqueta de metadiscurso. A través de estos el autor se hace presente en su texto. En este trabajo se analizarán algunos de estos procedimientos, concretamente construcciones con los verbos poder, caber, deber, haber y convenir más infinitivo, para demostrar su función atenuante en esta tipología textual. Para ello se han analizado 400 textos de cuatro disciplinas científicas.

PALABRAS CLAVE: artículo científico, atenuación, metadiscurso, directrices.
\end{abstract}

\begin{abstract}
Scientific discourse has its own and unique characteristics. Among them we can find a series of resources that have been labelled metadiscourse. By using metadiscourse the author of a text communicates with future readers. In this paper, we will analyse the resources used to mitigate in Spanish scientific articles. We will study the verbs poder, caber, deber, haber and convenir followed by the infinitive form of the main verb. To do so, we have used a corpus of 400 texts from four different disciplines.
\end{abstract}

KEY WORDS: research article, metadiscourse, mitigation, directives.

\section{INTRODUCCIÓN}

El artículo de investigación es un texto que se enmarca en el género científico y, dentro de esta categoría, pertenece a los "géneros de menor extensión” (Regueiro y Sáez 2013: 79). Se trata de breves publicaciones especializadas en las que se presenta una teoría desarro-

Para citar este artículo / To cite this article: Chao Parapar, Maikel (2018): Procedimientos de atenuación en los artículos de investigación científica: las directrices cognitivas en español. García Ramón, Amparo y Soler Bonafont, María Amparo (Eds.): ELUA: Estudios de atenuación en el discurso, Anexo IV, págs. 139-156.

Enlace / Link: http://dx.doi.org/10.14198/ELUA2018.Anexo4.08 
llada por un autor o grupo de autores cuyo objetivo será transmitir nuevos conocimientos y avances en uno de los campos del saber, así como persuadir y convencer al lector y a la comunidad científica de que esa es la más acertada. Por ende, en estos se aúnan las características de los textos expositivos $\mathrm{y}$, como se ha venido estudiando durante las últimas décadas, los argumentativos.

Durante mucho tiempo el discurso científico se ha visto caracterizado por las propiedades de objetividad, impersonalidad, claridad y precisión. La ausencia de marcas que hagan referencia al autor es uno de los elementos más repetidos en los diversos manuales de estilo de escritura científico-académica. Así, por ejemplo, en uno de los más actuales, podemos encontrar un consejo como el siguiente "lo adecuado para la escritura académica y profesional es que no aparezcan referencias al emisor ni al receptor, dado que este tipo de textos está muy alejado de la oralidad, la implicación personal y la subjetividad" (Montolío 2014: 451). Incluso se indica, unas líneas más adelante, que el hecho de reflejar a los participantes del acto comunicativo es muestra de inexperiencia.

Sin embargo, desde hace ya unas décadas, podemos encontrar estudios (en el ámbito anglosajón, sobre todo) en los que se analiza la presencia del autor de un texto científico con el objetivo de establecer una conexión con el futuro lector para conseguir que este comprenda el texto, la teoría que allí se expone y convencerlo de su viabilidad. Los diversos elementos lingüísticos empleados para crear esta relación autor-lector o autor-texto han sido agrupados bajo la etiqueta de metadiscurso. No obstante, este término no se utiliza en los estudios de análisis del discurso siempre con el mismo sentido. Para algunos autores bajo esta etiqueta solo se pueden agrupar aquellas expresiones que ayudan a estructurar el texto o que designan la propia acción del hablar o el escribir. Para otros, empero, como aquí veremos, el metadiscurso es mucho más amplio, más complejo y va mucho más allá del propio texto. Se trata de un mecanismo lingüístico necesario para que la comunicación escrita sea mucho más eficaz (Hyland 2010).

Agrupados en este mecanismo se encuentran muchos tipos de elementos lingüísticos con funciones completamente diferentes unos de otros. Por ello, algunos estudios han intentado clasificarlos en grupos que pudiesen abarcar las diversas funciones, así como las unidades lingüísticas de las que el autor se sirve para emplear el metadiscurso. Para nuestro estudio partimos de varias monografías publicadas por Ken Hyland, en particular la de Hyland y Tse (2004) y Hyland (2005), y de las observaciones realizadas tras el análisis de una selección de artículos extraídos de un corpus. Propondremos una clasificación del metadiscurso en español. De la tipología resultante, nos centraremos en el grupo llamado directrices y las analizaremos parar comprobar si estos elementos cumplen una función atenuante en los textos científicos que constituyen el corpus. De este modo, pretendemos establecer un primer acercamiento a estas construcciones, ver en qué contextos se dan, así como las diferentes funciones pragmáticas que pueden realizar. Los resultados de este análisis nos permitirán conocer las semejanzas y diferencias del empleo de estas construcciones en el corpus total, pero también comprender de qué modo se atenúa en las diversas ciencias aquí analizadas.

Tras el primer apartado, que sirve de introducción al estudio, en el segundo se delimitará conceptualmente el metadiscurso, sus tipos y categorías y en el tercero se expondrá el concepto de atenuación utilizado. En el cuarto apartado se expondrá la metodología empleada para el análisis del corpus. El quinto recogerá los resultados del análisis realizado, y, por último, el sexto apartado resumirá las principales conclusiones del estudio. 


\section{METADISCURSO: CONCEPTO Y PROPIEDADES}

\subsection{Estudios previos}

Como ya se ha dicho, en las últimas décadas se han realizado en el ámbito anglosajón numerosos estudios que indagan sobre los diferentes procedimientos por los cuales el autor de un texto científico se hace presente en su propio discurso. A pesar de que fue en 1959 cuando Zellig S. Harris acuña el término metadiscurso, no será hasta los años ochenta cuando comience a utilizarse para hacer referencia a aquellos elementos lingüísticos que no pertenecen al contenido proposicional de los diferentes enunciados que constituyen el texto. El metadiscurso fue definido en un comienzo como escritura sobre la escritura (Williams 1990 [1981]), y en esta teoría se englobaban todos los elementos que hiciesen referencia a la propia acción de escribir, así como a las diferentes partes en las que se divide el discurso. Curiosamente, este autor indica que la utilización de dichos elementos es un acto accidental que se produce involuntariamente durante el proceso comunicativo, como si el autor no tuviese voluntad a la hora de utilizarlos y se tratase de una parte del discurso sobre la que no tenemos control y que, por tanto, no podemos emplear en nuestro beneficio.

Partiendo de la explicación de Harris, aparece una nueva, aunque muy parecida, definición "discurso sobre el discurso o la comunicación sobre la comunicación" (Vande Koople 1985:83). Se define como aquella parte del discurso, ya hablado ya escrito, que no aporta nuevo contenido proposicional. Así, clasifica los diversos elementos en siete clases de funciones metadiscursivas (text connnectives, code glosses, illucotion markers, validity markers, narrators, attitude markers y commentaries) que ayudan a orientar al lector sobre diferentes aspectos relacionados con la redacción del texto o la fundamentación de su contenido.

El autor que más ha profundizado en la noción de metadiscurso ha sido el lingüista británico Ken Hyland, quien a través de un importante número de artículos ha perfilado y definido el concepto y ha investigado cómo está presente en el discurso académico. Para Hyland, el metadiscurso es una categoría funcional que se manifiesta a través de una serie de procedimientos lingüísticos por medio de los cuales el autor del texto organiza su discurso, se dirige al lector o expresa su opinión hacia lo escrito (cfr. Hyland 1998a, 2000, 2005, 2010; Hyland y Tse 2004).

Nos fijaremos principalmente en el artículo que Hyland escribió en colaboración con Tse (Hyland y Tse 2004) en el que realizan un replanteamiento del metadiscurso. Señalan las dificultades que plantea el estudio de los procedimientos metadiscursivos, lo que puede explicar la escasez de monografías sobre el tema. Una de las principales dificultades es la identificación de los elementos que desempeñan funciones metadiscursivas, ya que un mismo elemento puede ser metadiscursivo en unos contextos y no en otros; además, aun siendo metadiscursivo, puede pertenecer a diferentes subcategorías dependiendo del contexto en el que se enmarquen.

Para saber si un elemento del discurso desempeña una función metadiscursiva, los autores establecen una serie de criterios:

1. Retoman la característica básica que ya habían establecido Williams (1981) y Vande Koople (1985): los elementos deben ser externos al contenido proposicional. Sin embargo, indican que en muchos casos esta distinción es casi imposible, es decir, existen partículas metadiscursivas que además realizan una función dentro del contenido proposicional. Advierten, al mismo tiempo, que una división estricta 
puede relegar al metadiscurso a un mero comentario en lugar de reconocerle el puesto que este ocupa en el proceso comunicativo (Hyland y Tse 2004: 161).

2. La relación autor-lector-texto. Para los autores un texto no es solo el contenido científico que se expone, sino que se trata de un acto comunicativo y social en el que el autor depende de la futura opinión de los posibles lectores. Por lo tanto, el texto debe contener ciertos elementos que ayuden a crear la relación entre los participantes de la comunicación entre sí y la de estos con el propio texto. Lo que les lleva a concluir "we should see text as a process in which writers are simultaneously creating propositional content, interpersonal engagement, and the flow of text as they write, which means that their linguistic choices often perform more than one function" (Hyland y Tse 2004: 162).

3. La distinción de lo interno y lo externo. Para explicar este punto hacen referencia a los conectores textuales, en los que se puede observar claramente cuándo se establece una conexión en el propio discurso (interno) y cuándo la conexión es entre objetos o acciones pertenecientes al mundo real (externo) (Hyland y Tse 2004). Además, señalan que esta característica es crucial para poder diferenciar lo que pertenece al contenido proposicional de aquello que es propiamente metadiscursivo.

En el artículo al que estamos haciendo referencia, Hyland y Tse establecen una clasificación de los recursos de metadiscurso en dos grandes grupos: metadiscurso textual (interactive resource), aquel que establece la relación entre diversas partes de un texto, guiando al receptor durante su lectura, y un metadiscurso interpersonal (interactional resources), el que establece una relación con el lector, una relación que será crucial para que la teoría del autor del texto sea aceptada (Hyland y Tse 2004). Asimismo, cada categoría tiene diversos grupos en los que poder clasificar las partículas (Tabla 1).

En nuestro estudio nos fijaremos en el segundo grupo de recursos, los interaccionales, en el que según observamos en el cuadro hay cinco categorías: hedges, que se encargan de atenuar el contenido del texto; booster, cuya función es la de resaltar ciertas partes del discurso; attitude markers, que sirven parar reflejar la subjetividad del propio autor; engagement markers, que establecen una conexión directa con el lector; y self-mentions, que es el uso del pronombre con el que el autor se presentará a su audiencia.

\begin{tabular}{|c|c|c|}
\hline Category & Function & Examples \\
\hline Interactive resources & Help to guide reader through the text & \\
\hline Transitions & $\begin{array}{l}\text { express semantic } \\
\text { relation between main clauses }\end{array}$ & in addition / but / thus / and \\
\hline Frame markers & $\begin{array}{l}\text { refer to discourse acts, sequences, or } \\
\text { text stages }\end{array}$ & $\begin{array}{l}\text { finally / to conclude / my } \\
\text { purpose here is to }\end{array}$ \\
\hline Endophoric markers & $\begin{array}{l}\text { refer to information in } \\
\text { other parts of the text }\end{array}$ & $\begin{array}{l}\text { noted above / see Fig. / in } \\
\text { section } 2\end{array}$ \\
\hline Evidentials & $\begin{array}{l}\text { refer to source of information from } \\
\text { other texts }\end{array}$ & $\begin{array}{l}\text { according to } X /(Y, \\
1990) / Z \text { states }\end{array}$ \\
\hline Code glosses & $\begin{array}{l}\text { help readers grasp } \\
\text { functions of ideational material }\end{array}$ & $\begin{array}{l}\text { namely / e. g. / such as / in other } \\
\text { words }\end{array}$ \\
\hline Interactional resources & Involve the reader in the argument & \\
\hline
\end{tabular}




\begin{tabular}{|c|c|c|}
\hline Hedges & $\begin{array}{l}\text { withhold writer's full commitment } \\
\text { to proposition }\end{array}$ & might / perhaps / possible / about \\
\hline Boosters & $\begin{array}{l}\text { Emphasize force or } \\
\text { writer's certainty in proposition }\end{array}$ & in fact / definitely / it is clear that \\
\hline Attitude markers & $\begin{array}{l}\text { express writer's attitude to } \\
\text { proposition }\end{array}$ & $\begin{array}{l}\text { unfortunately / I agree / } \\
\text { surprisingly }\end{array}$ \\
\hline Engagement markers & $\begin{array}{l}\text { explicitly refer to or } \\
\text { build relationship with reader }\end{array}$ & $\begin{array}{l}\text { consider / note that / you can } \\
\text { see that }\end{array}$ \\
\hline Self-mentions & explicit reference to author(s) & I / we / my / our \\
\hline
\end{tabular}

Tabla 1. Cuadro de la clasificación de Hyland y Tse (tomado de Hyland y Tse 2004:169).

Esta clasificación ha sido perfeccionada y completada en estudios posteriores del propio Hyland. Así, en un artículo de 2005, dentro del grupo de los recursos interactivos, distingue entre stance, mecanismos que tienen como objetivo el texto o el propio autor; y engagement (Hyland 2005), recursos dirigidos al hipotético lector, tal como vemos en el esquema (Tabla 1).

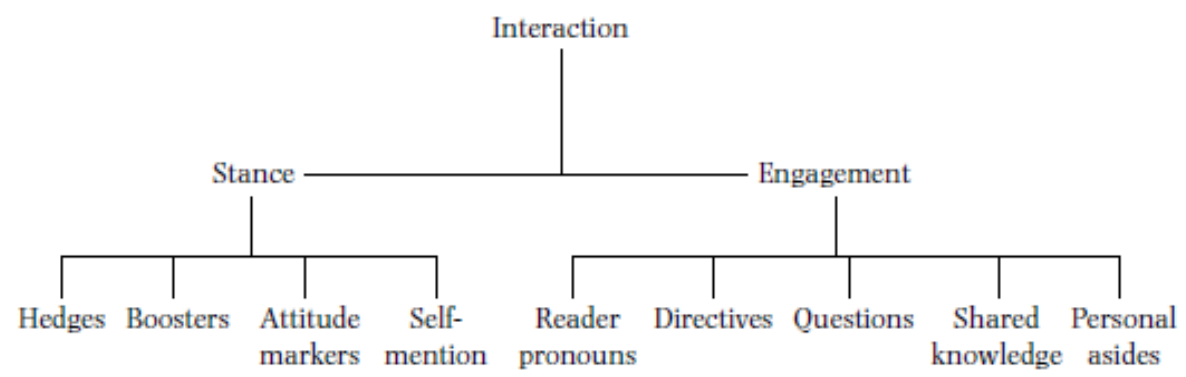

Imagen 1. Stance y Engagement (tomado de Hyland 2005: 177).

En la Imagen 1 observamos que se han creado cinco nuevas categorías: reader pronouns, que hacen mención del posible lector; directives, que establecen una orden; questions, preguntas que se hacen a la futura audiencia; shared knowledge, expresiones que establecen el conocimiento compartido; personal asides, pequeños apartes en los que se comparten opiniones o se aprovechan para hablar con más libertad (Hyland 2005).

\subsection{El metadiscurso en español}

En español la investigación sobre el discurso científico es amplia, aunque no tanto como en el ámbito anglosajón: Oliver del Olmo (2004), Morales, Cassany y González (2007), Morales y Cassany (2008), entre otros. En los trabajos que nos muestra la bibliografía, encontramos algunos que aplican la teoría de Hyland a textos científicos escritos en español. Por ejemplo, Beke (2005) analiza el uso de ciertas unidades con valores metadiscursivos en artículos de investigación en el campo de la Educación. Por su parte, Suau Jiménez (2010) desarrolla un estudio que trata de aplicar la teoría de Hyland y Tse (2004) a los textos del ámbito de la economía y la empresa. 
En cuanto a las unidades lingüísticas con función metadiscursiva, encontramos que la mayor parte de las que se enmarcan dentro de la función textual han sido ampliamente estudiadas, al corresponder muchas de ellas a marcadores discursivos, que como es sabido han recibido una gran atención en las últimas décadas. Por el contrario, las unidades que corresponden al segundo tipo de metadiscurso, el interpersonal, han sido menos estudiadas, sobre todo en lo que al discurso científico se refiere, razón por la que abordamos su estudio, con el interés de contribuir a este campo.

Un primer acercamiento a los textos que constituyen el corpus para el estudio que expongo en el presente artículo, me ha permitido constatar que las categorías de los recursos metadiscursivos interaccionales propuestos por Hyland (2005) (vid. supra Imagen 1) no se ajustan exactamente a los recursos que se utilizan en el español para expresar las relaciones del autor con su texto o con el interlocutor.

Como ya hacían Hyland y Tse (2004), diferenciamos también dos tipos de metadiscurso. En primer lugar, tenemos el metadiscurso textual, es decir, aquel que tiene como función principal la de establecer una jerarquía, una división o una conexión entre diversas partes de un mismo texto o la de poner en relación diversos textos; en el ejemplo 1, observamos como el segmento en cursiva sirve para poner en relación el apartado en el que se halla el lector con el siguiente, adelantando o introduciendo el tema. En segundo lugar, está el metadiscurso interpersonal, es decir, aquel que tiene como función principal la de establecer un vínculo entre los participantes del proceso comunicativo entre sí o entre estos (autor y lector) con el propio contenido proposicional; en el ejemplo 2 el elemento resaltado se encuadra entre los recursos metadiscursivos interpersonales porque, mediante su empleo, el autor manifiesta su posición respecto a la idea expresada al presentarla como posible.

(1) En el siguiente apartado se tratará el tema de la mediación docente en los procesos iniciales y formales del aprendizaje de la lectoescritura, ya que las autoras de este artículo consideramos que los docentes y las docentes tienen un papel protagónico para llevar a la práctica de manera exitosa este nuevo programa de estudios. (Educación)

(2) La prueba es el proceso o producto conseguido, pero para aquellas personas que se esfuerzan y no logran lo esperado, el reconocimiento extrínseco del esfuerzo puede llegar a estimular la autoeficacia, si el sujeto que lo reconoce le da valía al esfuerzo subordinando el logro a una jerarquía menor, haciéndole ver que los logros son la mayoría de las veces estáticos y el valor del esfuerzo no, lo que podría generar que las expectativas y la atención viren hacia el mérito del esfuerzo y no del logro. (Educación)

Para la creación de nuestra propuesta hemos tenido en cuenta la última clasificación de Hyland (2005) en la que propone que el metadiscurso interpersonal se divide en dos grandes grupos: stance y engagement, y a su vez cada uno de estos grupos tiene una posterior subdivisión, por lo que al final resulta una subclasificación en un amplio número de subcategorías que nos permite un punto de referencia más adecuado para el estudio de los recursos metadiscursivos del español. No obstante, como comprobaremos, en nuestra lengua, el funcionamiento de estos recursos no siempre coincide con lo que Hyland explica para el inglés. Así, por ejemplo, algunos de los recursos que en inglés serían considerados propiamente dirigidos al lector, en español pueden tener también como objetivo al propio autor del texto o, en algunos casos, a ambos participantes del discurso. 
El modelo que proponemos en la Tabla 2 para el metadiscurso interpersonal en español, realizado a partir de un corpus de textos científicos, contiene seis grandes grupos: atenuadores, intensificadores, evaluadores, marcadores de interacción, marcadores personales y referenciales. Con estas categorías se intenta abarcar la gran mayoría de unidades que puedan realizar una función metadiscursiva interpersonal.

\begin{tabular}{|c|c|c|c|}
\hline ATENUADORES & \multicolumn{3}{|c|}{$\begin{array}{l}\text { "Esto podría deberse a que la salud de los pacientes con más edad se encuentra } \\
\text { muy deteriorada, haciéndolos no aptos para un trasplante" (Medicina) }\end{array}$} \\
\hline INTENSIFICADORES & \multicolumn{3}{|c|}{$\begin{array}{l}\text { "Evidentemente, también han entrado palabras del inglés que en su original } \\
\text { contienen el sonido palatal sordo y que no necesariamente entran por la } \\
\text { emigración" (Lingüística y Literatura) }\end{array}$} \\
\hline EVALUADORES & \multicolumn{3}{|c|}{$\begin{array}{l}\text { "Es dificil saber el porcentaje de pacientes que sufre un duelo complicado ante } \\
\text { la psicosis" (Psicología y Psiquiatría) }\end{array}$} \\
\hline \multirow{8}{*}{$\begin{array}{l}\text { MARCADORES DE } \\
\text { INTERACCIÓN }\end{array}$} & \multirow{3}{*}{ Directrices } & Textuales & $\begin{array}{l}\text { “... puede ser visto como una realización } \\
\text { de una estructura jerárquica (vea Figura } \\
\text { 1)" (Matemáticas) }\end{array}$ \\
\hline & & Físicas & $\begin{array}{l}\text { "... luego se toma una alícuota de } 50 \mathrm{~mL} \\
\text { y [se] traslada a un balón de } 100 \mathrm{~mL} \text {..." } \\
\text { (Ingeniería) }\end{array}$ \\
\hline & & Cognitivas & $\begin{array}{l}\text { “.. hay que resaltar la importancia } \\
\text { de implementar una insensibilización } \\
\text { perfecta..." (Zoología y Veterinaria) }\end{array}$ \\
\hline & \multirow{3}{*}{ Acercamientos } & Apelaciones & $\begin{array}{l}\text { “... permitaseme traer a colación varios } \\
\text { casos en los cuales" (Derecho) }\end{array}$ \\
\hline & & $\begin{array}{l}\text { Plural } \\
\text { inclusivo }\end{array}$ & $\begin{array}{l}\text { "En los últimos años hemos asistido a una } \\
\text { nueva edición de las «guerras educativas» } \\
\text {.." (Derecho) }\end{array}$ \\
\hline & & Preguntas & $\begin{array}{l}\text { "¿qué poesía no es "palabra esencial” } \\
\text { o no se ocupa de la "esencia” de las } \\
\text { cosas?" (Lingüística y Literatura) }\end{array}$ \\
\hline & \multirow[t]{2}{*}{ Apartes } & Informativas & $\begin{array}{l}\text { "... se constituyeron en lugar de } \\
\text { resistencia (pasiva) de una población } \\
\text { abocada al abismo ..." (Historia y } \\
\text { Estudios Culturales) }\end{array}$ \\
\hline & & De opinión & $\begin{array}{l}\text { "(quizá por eso Lorca se refería a la } \\
\text { masturbación como "el sacrificio del } \\
\text { semen"...)" (Arte) }\end{array}$ \\
\hline \multirow{2}{*}{$\begin{array}{l}\text { MARCADORES } \\
\text { PERSONALES }\end{array}$} & \multicolumn{2}{|c|}{$\begin{array}{l}\text { De autor } \\
\text { o automenciones }\end{array}$} & $\begin{array}{l}\text { "Me parece conveniente partir de una } \\
\text { definición de lo que entendemos por } \\
\text { cultura..." (Ciencias Económicas) }\end{array}$ \\
\hline & \multicolumn{2}{|c|}{ De lector } & $\begin{array}{l}\text { "Tenéis (tenemos) el honor y el placer } \\
\text { de escribir en un folio en blanco..." } \\
\text { (Educación) }\end{array}$ \\
\hline REFERENCIALES & \multicolumn{3}{|c|}{$\begin{array}{l}\text { "Algunos autores afirman que las mujeres con cáncer de mama más jóvenes } \\
\text { padecen a menudo distintos efectos secundarios que las mayores y enfrentan } \\
\text { diferentes desafíos que las pacientes posmenopáusicas, como esterilidad, } \\
\text { pérdida de la masa ósea, menopausia prematura y mayor fatiga" (Medicina) }\end{array}$} \\
\hline
\end{tabular}

Tabla 2. El metadiscurso en español. 
A continuación, explicaré cada una de las categorías y subcategorías presentadas en la anterior tabla. La primera categoría recibe el nombre de "atenuadores". En este grupo tienen cabida todos aquellos elementos cuya función es la de minimizar el impacto del decir o de lo dicho, acorde a lo establecido por Briz (1995). Muchos trabajos como los de Caffi (1999) o Albelda (2004) entienden la atenuación (y la intensificación) como un fenómeno escalar, es decir, presenta diversos grados. Es esta gradación la que permite que nos encontremos con elementos que funcionan principalmente como atenuadores, así como algunos que pertenecen principalmente a una subcategoría diferente, pero que además realizan una función secundaria de atenuación.

Intensificadores es el nombre que reciben los elementos clasificados en la segunda categoría. Estos son términos que se emplean para destacar o subrayar una parte del contenido proposicional (Hyland y Tse 2004). Como ocurría con la atenuación, la intensificación puede presentar diversos grados. Estas dos categorías muestran el compromiso que el autor está dispuesto a asumir con el contenido proposicional (Hyland y Tse 2004).

Aquellas partículas que se agrupan en la tercera categoría, que recibe el nombre de evaluadores, se utilizan para formular un juicio sobre una idea expresada en el texto. Es posible, además, que un segmento metadiscursivo presente una múltiple función. En este grupo es muy común la presencia de otras funciones metadiscursivas, es decir, la partícula que califica el argumento suele realizar, a su vez, una atenuación o una intensificación de lo dicho.

Marcadores de interacción es el nombre que recibe la cuarta categoría. En esta se recogen términos que establecen una relación más directa con el lector. Estos pueden acercarse al público lector en menor o mayor medida. Por ello, hemos tenido que establecer tres clases: directrices, acercamientos y apartes.

Las directrices son elementos lingüísticos que ordenan al lector realizar o no una acción determinada por el autor (Hyland 2002). Son unidades complejas que implican estrategias retóricas en las que un autor manipula la relación con sus lectores e indica la manera en la que estos deben entender o seguir el texto, por lo que han sido vistas como amenazas hacia la relación autor-lector (Hyland 2002). Es por ello que se suelen utilizar en estructuras impersonales como estrategia de atenuación para no romper la relación de igualdad establecida entre los participantes de la comunicación científica.

A su vez, hemos dividido las directrices en tres clases, siguiendo el modelo que ya presentaba Hyland (2002): las directrices textuales, que hacen referencia bien a partes del mismo texto o a otros textos; las directrices físicas, muy comunes en las ciencias naturales, en las que se ordena realizar una acción fuera de la lectura del texto; las directrices cognitivas, que indican al lector qué proceso mental ha de realizar sobre el texto que está leyendo.

Los acercamientos tienen como objetivo unir al autor y al lector durante la lectura, estableciendo un contacto mucho más directo de lo que se considera apropiado para el género discursivo que nos ocupa. Para establecer dicho contacto, pueden utilizarse tres estrategias: las apelaciones, aunque poco comunes, se emplean para ganar la confianza del lector al tiempo que se formula un argumento que favorece la teoría del autor; el plural inclusivo, que "incorpora al destinatario en la mención que se hace" (NGLE: 1174), establece un nivel de confianza mayor al considerar al lector como parte del ámbito científico; finalmente, las preguntas suelen emplearse retóricamente o con el objetivo de focalizar la atención del lector en el nuevo argumento que se va a presentar. 
Los apartes son elementos que están escritos entre paréntesis, corchetes o guiones. Contienen opiniones o matizaciones sobre lo dicho en el contenido proposicional. En algunos casos, aunque no es muy común, se emiten juicios de valor o se realizan preguntas al lector. Debemos indicar que no podemos considerar como apartes las aclaraciones escritas entre paréntesis empleadas para explicar el significado de un término o las palabras que componen una sigla. Sin embargo, hay ciertos elementos informativos que sí deberían ser considerados en esta categoría. Por ello, hemos decidido establecer dos grupos: los apartes informativos, en los que se incluye información secundaria, pero necesaria para que el lector acepte la teoría del autor; los apartes de opinión, en los que se recogen aquellos elementos que no incluyen contenido proposicional de menos importancia, sino que son utilizados para crear un vínculo con el lector.

La quinta gran categoría es la de los marcadores personales, que engloban lo que Hyland (2005) había denominado selfmentions y reader pronouns. Se trata de marcas explícitas de la presencia del autor o del lector, ya sea mediante un pronombre personal, la flexión del verbo, un determinante posesivo...

Finalmente, hemos creado un grupo denominado referenciales en los que se incluyen aquellas expresiones que no hacen referencia a un estudio o autor en particular, sino que se utilizan para generalizar con el fin de persuadir al lector de que cierto argumento es sólido. Por tanto, diferenciamos aquellas que son metadiscursivas textuales, que sí unen dos o más textos mediante las convenciones bibliográficas, de las metadiscursivas interpersonales, cuya función es crear en la mente del lector la idea de que aquello que se va a comentar está ya bien arraigado en la comunidad científica.

Mediante el uso de todas estas categorías el autor puede conseguir guiar al lector en el discurso, así como persuadirlo de que la teoría expuesta es la correcta. Esta orientación, como ya indicaban Hyland y Tse (2004), es crucial para el autor, ya que el lector está interpretando el texto y un uso apropiado del discurso puede llegar a impedir que se posicione en contra de lo que se expone.

\section{ATENUACIÓN}

Los primeros estudios sobre la atenuación surgen en los años setenta (Lakoff 1972, Lysvag 1975) y durante las últimas décadas se ha publicado un extenso número de trabajos sobre este fenómeno, entre los que destacan Briz (1995) y Caffi $(1999,2007)$.

Se entiende por atenuación una estrategia pragmática con la que se minimiza la fuerza ilocutiva de los actos de habla y que pretende reducir el efecto de lo dicho o lo hecho (Albelda y Cestero 2011). Briz (1995) divide la atenuación en dos tipos: la atenuación semántico-pragmática (la que incide sobre lo dicho) y la atenuación pragmática (aquella que incide sobre el decir). Por su parte, Caffi $(1990,2007)$ divide los elementos atenuadores en tres clases: bushes, hedges y shields.

La atenuación se ha estudiado principalmente en el registro oral informal (véanse las publicaciones del grupo Val.Es.Co. y más recientemente del grupo Es.Var.Atenuación), si bien actualmente contamos con trabajos en los que se estudia en registros formales, tanto orales (Villalba 2012) como escritos. Entre estos últimos, destacamos varios trabajos sobre la atenuación en el ámbito académico-científico como Oliver del Olmo (2004), Morales, Cassany y González (2007), Morales y Cassany (2008), Morales (2011) y Acín (2016, en 
prensa). Nuestro estudio se encuadra en este mismo ámbito y se centra en el análisis de diversos procedimientos atenuadores utilizados en artículos de investigación pertenecientes a varias ciencias, lo que nos permitirá observar, entre otros aspectos, las diferencias entre ellas en cuanto al uso de tales procedimientos.

\section{METODOLOGÍA}

El corpus que hemos empleado para la realización de este estudio está compuesto por un total de 400 artículos de investigación científica de cuatro disciplinas científicas, es decir, se ha trabajado con 100 textos de cada una de las disciplinas seleccionadas para este trabajo: Educación, Ingeniería, Medicina y Piscología. Dichos textos han sido tomados de la web Red Iberoamericana de Innovación y Conocimiento Científico (REDIB), han sido publicados entre los años 2000 y 2016 y su extensión es variada, dependiendo de la rama a la que pertenezcan.

La comunicación en los artículos de investigación tiene un evidente carácter distanciado (Teberosky 2007), es decir, el autor pretende alejarse del texto. Sin embargo, no puede olvidarse del receptor, al cual debe guiar durante el proceso de lectura. Para conseguirlo debe posicionarse en el papel de lector para asegurarse de que la lectura del texto sea no solo la adecuada, sino también una lectura que favorezca la aceptación del contenido. Para ello se emplea el metadiscurso, mecanismo que analizamos en este corpus. Entre los procedimientos se encuentra la atenuación, un recurso empleado con el objetivo de minimizar el efecto de lo afirmado (Albelda 2010). Pero los elementos metadiscursivos cambian no solo de un contexto a otro, sino también de una comunidad científica a otra y de un autor a otro. Por eso, este corpus nos permitirá observar si el uso de los procedimientos atenuantes que aquí estudiamos se da de modo equitativo en las diferentes disciplinas o si, por el contrario, el perfil del investigador o del público lector puede establecer contrastes entre estas ciencias.

Una de las principales dificultades que nos hemos encontrado en el análisis es el reconocimiento de los elementos atenuantes; no todos los casos son tan fácilmente reconocibles como el ejemplo 3, en el que nos encontramos un adverbio con función atenuante. Por el contrario, el fragmento destacado en el ejemplo 4 es tanto un elemento metadiscursivo como una parte del contenido proposicional, es decir, no se presenta una distinción clara entre ambas funciones.

(3) Normalmente los defectos establecen mecanismos de falla más prematuros a partir de los cuales se desarrolla un eventual mayor daño. (Ingeniería)

(4) Es imprescindible insistir en la importancia de la identificación de problemas en los aspectos funcionales y emocionales de las personas con demencia así como la relación entre estas variables y la $\mathrm{CdV}$, mediante la realización de más estudios que identifiquen prioridades en la intervención, diseñen y propongan programas de intervención específicos y evalúen su efectividad. (Educación)

Como ya hemos dicho, en el primer ejemplo el adverbio normalmente tiene una clara función atenuadora, el autor evita comprometerse con la afirmación que va a realizar. Sin embargo, en el segundo caso, el conjunto es imprescindible insistir realiza una triple fun- 
ción: en primer lugar, sería una directriz cognitiva dirigida a los propios autores, pero, a su vez, también contiene un elemento claramente intensificador (imprescindible) que constituye una evaluación de la actividad que deben realizar los interlocutores.

Para el presente estudio hemos realizado una selección de elementos, a partir de la clasificación propuesta en el cuadro (Tabla 1), que según nuestro análisis desempeñan una función atenuadora en el discurso científico. Nos hemos fijado en una serie de verbos que los escritores de este tipo de textos utilizan con frecuencia: el verbo poder, de uso frecuentísimo, y otros que se podrían encuadrar en el grupo que he llamado directrices cognitivas (cfr. supra): deber, haber, caber y convenir, todos ellos en construcciones con infinitivo.

El paso siguiente ha sido la búsqueda de estos verbos en el corpus con el programa informático AntConc. Obtenidos los resultados, hemos leído cuidadosamente las concordancias surgidas en las diferentes búsquedas para descartar los que no tenían función atenuante de los que sí. Posteriormente, se ha calculado la frecuencia de aparición, lo que nos ha permitido comparar el uso de estos elementos en las diversas ciencias que componen los subcorpus.

\section{RESULTADOS DEL ANÁLISIS}

El análisis del corpus nos permite afirmar que, en mayor o en menor medida, la atenuación es empleada en cada una de las ciencias que hemos estudiado. Las unidades que hemos seleccionado ayudan al autor a exponer sus argumentos de dos modos que le ayudarán a guiar al lector durante la lectura del texto sin la necesidad de involucrarse directamente con el contenido proposicional: la relativización y la desagentivación.

Con el uso de la relativización un autor minimiza su compromiso con la afirmación empleada en su argumentación, ya que la presenta como vaga o imprecisa (Briz y Albelda 2013). En este caso, hemos considerado el verbo poder como representante de esta estrategia en los artículos de investigación científica. Como veremos, su uso es extenso y su función atenuadora presentará grados dependiendo del contexto en el que se aplique.

La desagentivación, u ocultación del yo, es uno de los fenómenos más empleados en el lenguaje científico debido a la necesidad de mostrar un estudio objetivo en el que no haya presencia de un agente que realiza el trabajo. Para desaparecer el autor recurre al uso de estructuras como la tercera persona de singular o la primera de plural. Como hemos expuesto en el aparatado anterior, consideramos estudiar aquí cuatro directrices cognitivas que, por su significado o por la estructura en la que son empleadas, tienen también función atenuante: haber, deber, caber, convenir + infinitivo.

\subsection{Perífrasis verbal poder + infinitivo}

Gómez Torrego, en su capítulo de la Gramática descriptiva de la lengua española, indica que "existen dos verbos poder seguidos de infinitivo: uno, el de 'capacitación', que no sería auxiliar de perífrasis verbal; el otro, el de 'posibilidad', que sí sería un verdadero auxiliar" (Gómez Torrego 1999: 3359). Sin embargo, tanto en esta obra como en la Nueva gramática de la lengua española ambas construcciones se consideran perifrásticas. Debido a esto, aquí también hemos decidido tratarlas como perífrasis verbales de infinitivo.

El caso de la perífrasis con valor epistémico de conjetura se identifica por la posibilidad de sustituirla por las expresiones semilexicalizadas puede que y puede ser que o por los 
adverbios probablemente y posiblemente seguidos del presente de subjuntivo. Esta muestra una distancia con el contenido proposicional cuyo fin es el de proteger la imagen del autor. Dado que los resultados de una investigación son producto de un contexto y unos procedimientos determinados, con el fin de evitar posibles contradicciones en futuros estudios, el autor no realiza una afirmación concluyente.

(5) Esta variación podría considerarse poco significativa en términos porcentuales; sin embargo, debido a la magnitud de este tipo de proyectos, esta leve variación se amplifica en términos absolutos a medida que la cantidad de unidades de vivienda incrementa. (Ingeniería)

(6) Este marco podría explicar comportamientos como reprimir o negar la orientación homosexual, el llevar una doble vida o la ocultación de la orientación sexual. En este sentido, la ocultación puede conllevar impactos psicológicos negativos como un elevado nivel de ansiedad, un estado de alerta constante y un sentimiento de aislamiento y malestar por el encubrimiento de la identidad. (Psicología y Psiquiatría)

(7) Hemos de señalar que, cuando la investigación-acción se lleva a cabo en la clase con suficiente profundidad, puede incrementar la conciencia de los profesores sobre sus "implicaciones políticas" y, por tanto, realza su capacidad colectiva para influir sobre la puesta en práctica de cambios estructurales, tanto en las escuelas como en el sistema educativo. (Educación)

Cuando se utiliza la perífrasis modal radical, que denota capacidad, facultad o habilidad, nos encontramos con un proceso de desagentivación. En este caso el autor busca un tercer elemento que le permita afirmar sin la necesidad de involucrarse personalmente.

(8) A partir de los resultados del presente estudio, podemos resaltar la importancia de trabajar las competencias emocionales y la toma responsable de decisiones en los preadolescentes a partir de la ee en el seno familiar, que pudiera prevenir que los chicos caigan en situaciones de riesgo que afecten su salud mental y física. (Educación)

(9) Basados en nuestros resultados, podemos concluir que en nuestra población las mujeres consultan más frecuentemente que los hombres por acné, sobre todo aquellas con exposición social frecuente, que las formas graves de acné se ven con mayor frecuencia en jóvenes y en el sexo masculino y que las mujeres mayores de 25 años presentan formas leves a moderadas de la afección. (Medicina)

(10) Con estos resultados podemos sugerir que tanto las inferencias emocionales con valencia positiva como negativa se activan on-line (véase Figura 1). (Psicología y Psiquiatría)

Debemos indicar que este es el recurso más utilizado de todos los que aquí estudiamos, pues presenta una frecuencia de casi 21 casos cada 10000 palabras. Además, nuestro análisis muestra que la única disciplina en la que se puede observar una gran diferencia respecto a las demás es la Ingeniería, cuya frecuencia no alcanza los 3 casos por 10000 palabras.

Debido a que la perífrasis de conjetura puede ser empleada en un rango mayor de contextos ya que no depende de la presencia de un tercer elemento en el discurso, es normal que se dé la diferencia que podemos apreciar en la Gráfica 1. 


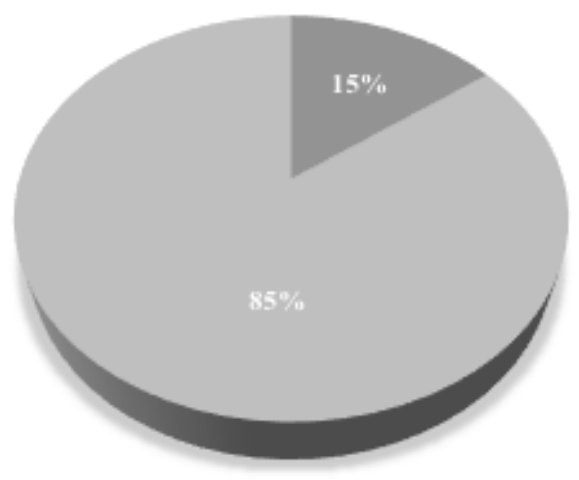

EDeóntico E Epistémico

Gráfica 1. Comparación poder.

\subsection{Directrices atenuantes}

\subsubsection{Deber + infinitivo}

Los valores semánticos habituales de esta perífrasis son los de obligación activa, necesidad y obligación pasiva (Gómez Torrego 1999). En nuestro corpus solo hemos estudiado el primero de ellos, ya que presenta sujetos agentes y, por tanto, el autor debe recurrir a la atenuación para que la orden que expresa no amenace la igualdad preestablecida en esta tipología textual.

En los siguientes ejemplos, el verbo deber se ha conjugado en primera persona de plural con el objetivo de ocultar el yo agente. No podemos establecer que se trate de un plural de autor o un plural inclusivo porque, dependiendo del contexto, el autor se escuda en uno (ejemplo 11) o en otro (ejemplo 12).

(11) Además debemos poner especial interés, como hemos observado en nuestro estudio, en el caso de parte de la población inmigrante que además de tener un fototipo cutáneo más oscuro y mayor déficit de ingestión de calcio, tienen un estilo de vestir tradicional que cubre gran parte del cuerpo debido a sus principios étnicos y religiosos, lo que puede resultar en una restricción más acentuada de la síntesis de vitamina D4-5,17,33,34. (Medicina)

(12) Debemos trabajarlo intensamente con propuestas didácticas útiles y eficaces para la práctica en ejercicio de la profesión. (Educación)

Además, también se utiliza en construcciones pasivas reflejas cuando el sujeto no es humano. Como vemos en los ejemplos, se utiliza la desagentivación para atenuar la orden que el autor da al lector para que siga el hilo argumental.

(13) Además de estos ajustes, se debe considerar la opción de operación de una línea larga y cargada (parámetros que dependen mucho de la configuración de la red), para solventar esta necesidad se cuenta con la característica de discriminación de carga. (Ingeniería) 
(14) Se debe indagar cuáles son sus atributos y significados, los cuales tienen como referente el intercambio cultural y la experiencia subjetiva, como posibilidad de re-significación y apropiación de una realidad simbólicamente pre-estructurada. (Psicología y Psiquiatría)

Nos encontramos ante una construcción bastante utilizada que llega a presentar 1,5 casos cada 10000 palabras. Cabe resaltar que son los artículos de Ingeniería los que presentan una menor frecuencia tanto en esta construcción como en las anteriores o en las que siguen a este apartado. Son, por tanto, uno de los recursos atenuantes menos productivos en el lenguaje académico de los ingenieros.

\subsubsection{Haber que + infinitivo}

Esta perífrasis modal de obligación tiene como rasgo fundamental su carácter encubridor del actor, sea agente o paciente. Es normal pues, que los infinitivos con los que se combina pertenezcan a verbos "que solo pueden llevar sujetos animados semánticamente actores fuera de la perífrasis" (Gómez Torrego 1999:3357). Se trata de una estructura impersonal que presenta el siguiente argumento como una obligación que el yo autor no puede evadir y que le permite ocultarse mientras introduce una información que favorecerá su argumentación.

(15) Por último, hay que señalar la contribución de Alemany y Lara (2010) quienes diseñaron y validaron una nueva escala de actitudes hacia las matemáticas para estudiantes de secundaria compuesta por 37 ítems. (Educación)

(16) Hay que tener en cuenta que estos hechos se presentaron con un evento que fue la octava parte del sismo de diseño; lo cual es un escenario preocupante, per se, ya que de acuerdo con la normativa vigente para este tipo de evento se supone que no debería haber pasado absolutamente nada. (Ingeniería)

En comparación con las perífrasis que ya hemos visto en anteriores apartados, este no es uno de los recursos más empleados. Presenta una frecuencia menor de 0.5 casos cada 10000 palabras en la ciencia que más lo emplea (Educación) y no se observa gran diferencia frente a la frecuencia que han presentado las otras disciplinas.

\subsubsection{Caber + infinitivo}

Por su significado esta construcción se utiliza para añadir al contenido proposicional más información que puede no ser estrictamente necesaria pero que ayudará al lector a comprender el hilo argumental del texto, así como al encubrimiento del yo. Como podemos ver en los ejemplos, se conjuga en tercera persona del singular

(17) Cabe señalar que para calibrar el modelo biológico fue importante determinar de manera confiable el caudal de purga de lodos (QWAS), ya que de este depende el tiempo medio de retención celular $(\theta \mathrm{C})$ en los reactores, lo cual se realizó satisfactoriamente a través del balance de los ISS y utilizando el software GPS-X para la simulación de escenarios de optimización. (Ingeniería) 
(18) Cabe decir que al incorporar estas herramientas de las TIC en el curso de fonética, se fomentó un aprendizaje más personalizado e individualizado en el estudiantado, pues al disponer de los contenidos desarrollados en la clase en la plataforma, cada estudiante pudo retomar los temas, consultar las nuevas fuentes de la información según su necesidad y también progresar de acuerdo con su ritmo de aprendizaje, y así plantear las preguntas al docente de algún punto que todavía generaba dudas. (Educación)

Tras el análisis del corpus, hemos podido observar que esta construcción presenta una frecuencia bastante amplia, llegando casi a 1,4 casos cada 10000 palabras. Como ocurría en casos anteriores, Ingeniería es la ciencia en la que menos se emplea con una frecuencia de 0.34 casos. Además, debemos indicar que esta construcción presenta la mayor diferencia de uso entre la disciplina que más lo emplea (Psicología) y la que menos (Ingeniería).

\subsubsection{Convenir + infinitivo}

Como la construcción verbal anterior, esta se utiliza para añadir información adicional al contenido proposicional. En este caso, por su significado, resulta evidente que a quien le conviene que el lector tenga en cuenta lo que sigue a este verbo es al yo autor. Por tanto, para atenuar su presencia en el discurso, se conjuga el verbo en tercera persona del singular.

(19) Conviene, antes de ir más lejos en este análisis, preguntarse si la variación en el número de diferencias observadas significa o no una polarización de las respuestas en relación a la proporción media teórica. (Psicología y Psiquiatría)

(20) En cualquier caso, a la luz de estos resultados, conviene llamar la atención sobre el elevado riesgo de LT en nuestro medio en la población de más edad. (Medicina)

Es el recurso menos empleado, con 0.1889 casos cada 10000 palabras en la disciplina que más frecuencia presenta (Educación) y un 0.0211 en la que menor uso se ha registrado (Medicina).

\section{CONCLUSIONES}

En este trabajo hemos analizado diversos procedimientos atenuantes en el discurso científico, en concreto, en los artículos de investigación científica. Para ello hemos partido del concepto de atenuación propuesto por el grupo de investigación Es.Var.Atenuación y de los estudios sobre el metadiscurso realizados por Hyland (1998a, 1998b, 2000, 2005, 2010; Hyland y Tse, 2004) con el fin de analizar las funciones de diversos verbos en un corpus de 400 textos pertenecientes a varias ramas de conocimiento (Educación, Ingeniería, Medicina, Psicología y Psiquiatría). Como hemos visto, nos hemos encontrado con el verbo poder, cuya función atenuante es la de relativizar lo expresado, y con cuatro verbos (deber, haber, caber y convenir) con doble función, enunciar una orden, ya sea al público lector ya al propio autor, y atenuar la presencia del yo autor (Gráfica 2).

En la Gráfica 2 observamos la diferencia de frecuencia de uso de los recursos de atenuación que hemos estudiado. Casi un $70 \%$ corresponde al verbo poder, mientras el 30 $\%$ restante se reserva para el empleo de las directrices. Esto nos permite concluir que las 
construcciones atenuantes más productivas son las construidas con la primera perífrasis. Probablemente sea debido a que verbos como convenir muestran una mayor influencia del yo autor en el lector, mientras que poder puede ser empleado con una construcción impersonal o con el plural de modestia, lo cual ayuda a difuminar la presencia del autor del discurso científico y a fortalecer la imagen de impersonalidad y objetividad que todavía son las características más señaladas de esta tipología textual.

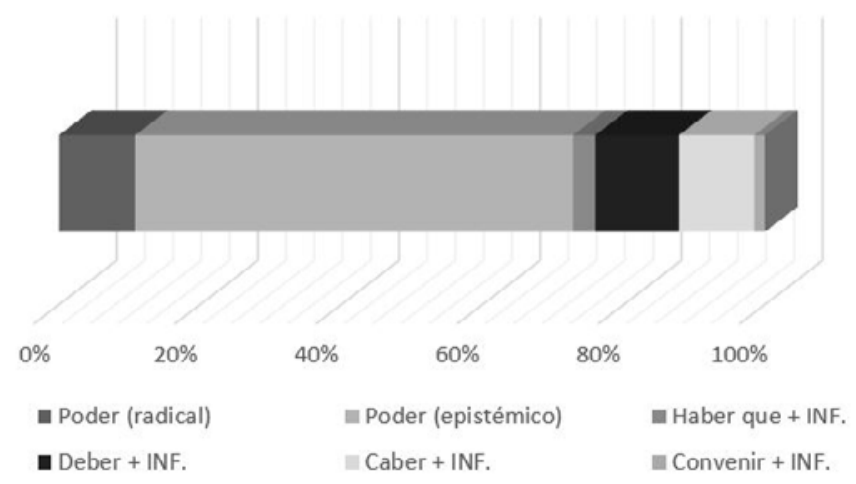

Gráfica 2. Uso de la atenuación.

Sí podemos afirmar, tras el análisis realizado, que los artículos pertenecientes a Ingeniería presentan un menor uso de procedimientos atenuantes verbales. Seguramente porque en las disciplinas técnicas hay una mayor insistencia en que el texto se presente como una verdad universal, un texto que se crea solo por la transmisión de nuevos datos en un campo determinado. Sin embargo, sí puede observarse en ciencias como Psicología o Educación una mayor presencia del autor en el texto.

Concluimos, por tanto, que nuestro trabajo ha pretendido ser una contribución al estudio de los textos científicos escritos en español, concretamente nos hemos fijado en el empleo que hacen sus autores de unas unidades lingüísticas que hemos demostrado que desempeñan una función de atenuación, entendida esta como estrategia pragmática al servicio del productor textual. Estudios posteriores, con corpus más amplios y con análisis de más unidades nos permitirán extraer conclusiones más sólidas en torno a la descripción del discurso científico en español.

\section{Referencias bibliográficas}

Acín, E. (2016). "La atenuación en las "Conclusiones" de las tesis de doctorado", Textos en proceso, 2 (1), pp. 1-24.

Acín, E. (2017). "Atenuación y discurso académico: las "Conclusiones" de la tesis de doctorado", Lingüística Española Actual, 39 (2), pp. 153-175.

Albelda, M. (2004). “La escalaridad de la intensificación”, Interlingüística, 15, pp. 105-114.

Albelda, M. (2010). “Cómo se reconoce la atenuación? Una aproximación metodológica basada en el español peninsular hablado”. En Orletti, F. y L. Mariottini (eds). (Des)cortesía en español. Espacios teóricos y metodológicos para su estudio. Roma: Università Roma Tre y Programa EDICE, pp. 41-70. 
Albelda, M. y A.M. Cestero (2011). "De nuevo, sobre los procedimientos de atenuación lingüística", Español Actual, 96, pp. 9-40.

Beke, R. (2005). "El metadiscurso interpersonal en artículos de investigación”, Signos, 38 (57), pp. 7-18.

Briz, A. (1995). "La atenuación en la conversación coloquial. Una categoría pragmática". En Cortés, L. (ed.). El español coloquial: Actas del I Simposio sobre análisis del discurso oral. Almería: Universidad de Almería. pp. 103-222.

Briz, A. y M. Albelda (2013). "Una propuesta teórica y metodología para el análisis de la atenuación lingüística en español y portugués. La base de un proyecto común (ES.POR.ATENUACIÓN)", Onomázein, 28, pp. 288-319.

Caffi, C. (1999). “On mitigation”, Journal of Pragmatics, 31, pp. 881-909.

Caffi, C. (2007). Mitigation. Oxford: Elsevier.

Gómez Torrego, L. (1999). "Los verbos auxiliares. Las perífrasis verbales de infinitivo". En Bosque, I. y V. Demonte (dirs.). Gramática descriptiva de la lengua española. Madrid: Espasa, pp. 33233390 .

Harris, Z. (1959). "The Transformational Model of Language Structure”, Anthropological Linguistics, 1 (1), pp. 27-29.

Hyland, K. (1998a). "Persuasion and context: The pragmatics of academic discourse", Journal of Pragmatics, 30, pp. 433-454.

Hyland, K. (1998b). "Boosting, hedging and the negotiation of academic knowledge, Text, 18, pp. 349-382.

Hyland, K. (2000). Disciplinary discourses: Social interactions in academic writing. Londres: Edward Arnold.

Hyland, K. (2002). "Directives: arguments and engagement in academic writing”, Applied Linguistics, 23, pp.215-239.

Hyland, K. (2005). Metadiscourse: Exploring interaction in writing. London: Longman.

Hyland, K. (2010). "Metadiscourse: Mapping Interactions in Academic Writing", Nordic Journal of English Studies, 9 (2), pp. 125-143.

Hyland, K. y P. Tse (2004). "Metadiscourse in Academic Writing: A Reappraisal”, Applied Linguistics, 25 (2), pp. 156-143.

Lakoff, G. (1972). "Hedges: a study in meaning criteria and the logic of fuzzy concepts", Journal of Philosophical Logics, 2, pp. 458-508.

Lysvåg, P. (1975). "Verbs of hedging”. En J. P. Kimball (ed.), Syntax and Semantics, 4. Nueva York: Academic Press, pp. 125-154.

Montolío, E. (dir.) (2014). Manual de escritura académica y profesional. Barcelona: Ariel.

Morales, O. A. (2011). Los géneros escritos de la Odontología hispanoamericana. Estructura retórica y estrategias de atenuación en artículos de investigación, casos clínicos y artículos de investigación. Estados Unidos: Editorial Academia Española.

Morales, O. y D. Cassany (2008). "Interpretaciones de la atenuación en artículos de revisión de Odontología”, Signos, 41 (67), pp. 299-328.

Morales, O., Cassany, D. y C. González (2008). "La atenuación en artículos de odontológicos publicados en español entre 1994 y 2004: Estudio exploratorio", Revista Ibérica, 14, pp. 33-58.

Oliver del Olmo, S. (2004). Análisis contrastivo español/inglés de la atenuación retórica en el discurso médico. El artículo de investigación en el caso clínico. Tesis doctoral, Universitat Pompeu Fabra.

Real Academia Española y Asociación de Academias de la Lengua Española (2009). Nueva gramática de la lengua española. Madrid: Espasa.

Regueiro, M. L. y D.M. Sáez (2013). El español académico. Guía práctica para la elaboración de textos académicos. Madrid: Arco/Libros.

Suau Jiménez, F. (2010). La traducción especializada (en inglés y español en géneros de economía y empresa). Madrid: Arco/Libros. 
Teberosky, A. (2012 [2007]). "El texto académico”. En Castelló, M. (coord.). Escribir y comunicarse en contextos científicos y académicos. Conocimientos y estrategias. Barcelona: Graó, pp. 17-46.

Vande Koople, W. J. (1985). "Some exploratory discourse on metadiscourse", College Composition and Communication, 36, pp. 82-93.

Villalba, C. (2012). "El valor atenuante de la impersonalidad en los juicios orales", Anuario de Lingüistica Hispánica, XXVIII, pp. 117-132.

Williams, J. M. (1990 [1981]). Style. Toward Clarity and Grace. Chicago: The University of Chicago Press. 